Leandro Liberino Silva ${ }^{\mathrm{a}}$

(iD) https://orcid.org/0000-0001-9346-2666

Leandro Portes Cury Lima ${ }^{b}$

(iD) https://orcid.org/0000-0002-2704-6338

Cristovam Chiaradia Barbosa

(iD) https://orcid.org/0000-0003-3982-3867

Aluisio Diniz Machadoc

(iD) https://orcid.org/0000-0002-1053-4589

Adriano Starling Moscic

(iD) https://orcid.org/0000-0002-9314-7436

Francisco das Chagas Lima e Silva ${ }^{d}$

(iD) https://orcid.org/0000-0002-6991-1465

Danielle Nunes Pinto Della Torre

(iD) https://orcid.org/0000-0002-8022-2505

Andréa Maria Silveira ${ }^{f}$

(iD) https://orcid.org/0000-0001-9629-9200

Ana Paula Scalia Carneiro ${ }^{\mathrm{e}}$

(iD) https://orcid.org/0000-0002-6220-6077

a Santa Casa de Belo Horizonte, Centro de Diagnóstico e Tratamento. Belo

Horizonte, MG, Brasil.

${ }^{\text {b} F a c u l d a d e ~ d e ~ C i e ̂ n c i a s ~ M e ́ d i c a s ~ d e ~}$

Minas Gerais, Departamento de Medicina

Ocupacional. Belo Horizonte, MG, Brasil.

'Justiça do Trabalho. Belo Horizonte,

MG, Brasil.

d Santa Casa de Belo Horizonte, Núcleo de Pós-Graduação e Pesquisa. Belo Horizonte, MG, Brasil.

e Universidade Federal de Minas Gerais, Hospital das Clínicas, Serviço Especializado em Saúde do Trabalhador (SEST). Belo Horizonte, MG, Brasil.

${ }^{f}$ Universidade Federal de Minas Gerais, Faculdade de Medicina, Departamento de Medicina Preventiva e Social. Belo Horizonte, MG, Brasil.

Contato:

Leandro Liberino Silva

E-mail:

liberino.silva80@gmail.com

Trabalho baseado em tese de doutorado de Leandro Liberino da Silva, intitulada Diagnóstico de silicose através de tomografia multidetectores em exmineiros de ouro com radiografias normais: quando indicá-la?, apresentada em 2017 ao Núcleo de Pós-Graduação e Pesquisa da Santa Casa de Belo Horizonte da Faculdade de Medicina da Universidade Federal de Minas Gerais (UFMG).

Os autores declaram que o trabalho não foi subvencionado e que não há conflitos de interesses.

Os autores informam que o trabalho não foi apresentado em reuniões científicas.

Recebido: 20/04/2017

Revisado: 23/07/2017

Aprovado: 13/08/2017

\section{Modificação do perfil da silicose na mineração subterrânea de ouro em Minas Gerais}

\author{
Changes on the silicosis profile in underground \\ gold mining in Minas Gerais, Brazil
}

\begin{abstract}
Resumo
Introdução: Minas Gerais é o estado brasileiro com maior registro de casos de silicose, sendo grande parte proveniente de casuísticas acumuladas das minerações de ouro. Objetivos: descrever e analisar temporalmente a ocorrência de silicose na mineração de ouro identificando fatores ocupacionais relacionados. Métodos: estudo transversal com 1.020 ex-mineiros da região de Nova Lima/MG, avaliados entre 1995 e 2011. Resultados: o diagnóstico de silicose foi confirmado em 19,7\% dos avaliados. Nenhum caso da doença foi identificado em indivíduos que trabalharam apenas na superfície. A prevalência no grupo que trabalhou até 5 anos no subterrâneo foi de 3,8\% e no grupo com mais de 20 anos de trabalho nesse local foi de $44,2 \%$. Os admitidos para trabalho subterrâneo até 1950 apresentaram prevalência de 57,9\%. Entre os admitidos após 1990, não houve registro de casos. Conclusão: verificou-se uma queda expressiva na ocorrência de silicose no período analisado. Uma vez que a doença é sabidamente dose-dependente, é esperado que a diminuição dos níveis de exposição, obtido pelas melhorias dos ambientes ocupacionais, tenha refletido nestes resultados. É fundamental que tais medidas continuem a ser adotadas na mineração e em outros ramos de atividade visando reduzir a ocorrência da doença.
\end{abstract}

Palavras-chave: pneumoconiose; silicose; doenças ocupacionais; mineração; ouro.

\begin{abstract}
Introduction: Minas Gerais is the Brazilian state with the highest record of silicosis cases, mostly from gold mining. Objectives: to describe and analyze the occurrence of silicosis in gold mining over time, identifying occupational related factors. Methods: cross-sectional study with 1.020 former miners from the region of Nova Lima, Minas Gerais, evaluated from 1995 to 2011. Results: silicosis diagnosis was confirmed in $19.7 \%$ of the miners evaluated. No cases were identified in individuals who worked only on the surface. The prevalence among those who worked underground was $3.8 \%$ for the group up to 5 years and $44,2 \%$ for the group that worked over 20 years. Those admitted to work underground up to 1950 had a prevalence of $57.9 \%$. Among those admitted after 1990, there were no cases recorded. Conclusion: we found an expressive decline in the silicosis occurrence in the period. Because silicosis is dose-dependent, it is expected that decrease in exposure levels was due to improvements in work environments. These measures must continue to be adopted in mining and in other branches of work activity to reduce the occurrence of silicosis.
\end{abstract}

Keywords: pneumoconiosis; silicosis; occupational diseases; mining; gold. 


\section{Introdução}

A silicose continua representando um sério problema de saúde pública em alguns países do mundo, especialmente naqueles pouco desenvolvidos ou em desenvolvimento ${ }^{1}$. São conhecidos diversos ramos de atividade que apresentam risco de exposição à poeira de sílica: mineração de metais, especialmente em subterrâneo; extração e beneficiamento de rochas; lapidação de quartzo e pedras preciosas; construção civil (perfuração de poços e túneis); jateamento de areia; metalurgia; entre outras ${ }^{2,3}$.Do ponto de vista fisiopatológico, a silicose tem início com a inalação de partículas de sílica que desencadeiam reação inflamatória cujo desfecho são focos de fibrose intersticial pulmonar sob a forma de pequenos nódulos regula$\mathrm{res}^{4,5}$. Estes podem coalescer e formar grandes massas fibróticas com o passar dos anos ${ }^{6}$. A exposição à sílica está associada ainda ao surgimento de doenças, como tuberculose, doenças autoimunes, doença pulmonar obstrutiva crônica e câncer de pulmão ${ }^{7-11}$.

Sabe-se que a silicose apresenta forte gradiente dose-resposta e, para que a exposição se torne efetivamente lesiva, os fatores mais importantes são a concentração de sílica livre na fração de poeira respirável e a duração da exposição ${ }^{12,13}$. Outros fatores importantes são o tamanho da partícula (risco maior para partículas menores de $10 \mu$ ), a natureza cristalina ou não cristalina da sílica, a sílica recém fraturada e a susceptibilidade individual às doenças provocadas pela sílica ${ }^{4,5,11,14}$.

O diagnóstico da doença é realizado pela integração da história clínica e ocupacional com achados de imagem, habitualmente radiológica, dentro de padrões determinados pela Organização Internacional do Trabalho ${ }^{15}$ (OIT). Nas imagens são visualizados micronódulos, eventualmente coalescentes, que habitualmente ocorrem nas regiões superiores e posteriores dos pulmões. Muitos estudos têm demonstrado que a tomografia computadorizada de alta resolução é mais acurada que a radiografia para identificar os nódulos silicóticos, bem como sua confluência e grandes opacidades ${ }^{16-18}$. Atualmente, a tomografia é um reconhecido método de imagem complementar à radiografia para a avaliação da exposição à sílica e da suspeita de silicose. Seus principais benefícios se dão no diagnóstico de casos iniciais da doença não manifestos na radiografia, bem como na avaliação de grandes opacidades em casos radiologicamente bem definidos $^{19}$. Os sintomas são relativamente tardios e posteriores às manifestações radiológicas e se caracterizam por dispneia, tosse e fraqueza.

No Brasil, a silicose é a pneumoconiose de mais alta prevalência. Estudo com dados de emprego no mercado formal em 2001 estimou como possivelmente expostos 976.939 trabalhadores (2,65\%), provavelmente expostos 2.404 .955 (6,52\%) e definitivamente expostos à sílica 2.065.929 (5,6\%), o que comparado a estudos internacionais colocava o Brasil entre os países com maior prevalência de exposição àquela poeira ${ }^{20}$.

Dos estados brasileiros, Minas Gerais apresenta o maior número de casos de silicose notificados, historicamente relacionados à extração e beneficiamento de minérios, particularmente casuísticas acumuladas da extração de ouro subterrânea na região de Nova Lima $^{21,22}$. No entanto, nos últimos anos, o perfil ocupacional dos doentes no estado vem se alterando com crescimento de casos entre trabalhadores do mercado informal ou de pequenas empresas, como lapidadores de pedras semipreciosas e trabalhadores na extração e beneficiamento de quartzo ${ }^{23-25}$.

Os primeiros estudos ${ }^{26,27}$ sobre a silicose no Brasil datam da década de 1940 e 1950 e foram realizados pelo Departamento Nacional da Produção Mineral. De acordo com eles, após a realização de radiografia de tórax em 908 trabalhadores que atuavam no subterrâneo, 304 (33,5\%) apresentaram critérios de diagnóstico de silicose ${ }^{27}$.

Em Nova Lima, a exploração do ouro teve início no século XVIII. A alta mortalidade decorrente das condições de trabalho penosas e insalubres motivou a vinda do primeiro médico para a cidade em 1838 e a construção de um hospital com 60 leitos em $1848^{28}$.

Na década de 1990 ocorreu uma intensa fiscalização nas minas por parte do Ministério do Trabalho, o qual verificou níveis elevados de poeira de sílica ${ }^{29}$. Ao mesmo tempo, o movimento com apoio do Sindicato dos Mineiros de Nova Lima culminou na indenizações de 694 ex-trabalhadores silicóticos. Diante desses fatos, a Assembleia Legislativa de Minas Gerais criou uma Comissão Parlamentar de Inquérito que determinou à empresa melhorar as condições de trabalho e incluir mais trabalhadores no processo indenizatório.

Assim, o objetivo do estudo foi descrever e analisar a ocorrência de silicose na região de Nova Lima/ MG e traçar o perfil clínico e ocupacional dos indivíduos avaliados em perícias médicas realizadas no período de 1995 a 2011.

\section{Métodos}

Trata-se de um estudo de desenho transversal cuja casuística foi composta por ex-trabalhadores de mineração de ouro da região de Nova Lima/MG, onde se localiza a principal empresa mineradora de ouro do país. Tais indivíduos eram provenientes 
de um universo estimado de 1.500 ex-trabalhadores que no início de década de 1990 abriram ações judiciais contra o ex-empregador pleiteando indenizações devido a possível adoecimento por silicose. Destes, aproximadamente 500 foram avaliados fora do âmbito judicial, em acordos coletivos, e não foram incluídos neste estudo. Os demais compuseram um grupo de 1.020 indivíduos avaliados em perícia médica, na Justiça Comum e na Justiça do Trabalho do município de Nova Lima, no período de 1995 a 2011. As avaliações foram realizadas por quatro dos autores deste estudo. Os reclamantes alegavam serem portadores de silicose adquirida no curso de seus trabalhos e solicitavam reparação civil.

Os dados utilizados foram obtidos a partir de prontuários padronizados das avaliações que continham histórico clínico, ocupacional, laudos de radiografia (RX) e tomografias computadorizadas de técnica de alta resolução (TCAR) de tórax. Esta última foi realizada em casos selecionados.

A dinâmica das avaliações se iniciava com a classificação das radiografias, que foram realizadas de forma independente por três leitores qualificados, sendo dois deles certificados de acordo com a padronização da OIT. No momento das classificações os leitores não conheciam os dados clínicos e ocupacionais dos indivíduos, portanto as leituras foram feitas de modo cego. As radiografias foram classificadas nas 12 subcategorias de profusão de pequenas opacidades segundo a classificação da OIT $^{15}$. Para análise foram utilizadas as medianas das classificações dos três leitores. A mediana é calculada como a medida central de acordo com a escala ordinal de 12 pontos da classificação. Tome-se como exemplo um exame com as classificações: 0/0; 0/1 e 1/0. A mediana será $0 / 1$. De acordo com a literatura, considera-se como caso de pneumoconiose, aquele cuja leitura é igual ou maior que $1 / 0$, desde que com história clínica e ocupacional compatíveis ${ }^{15}$.

A seguir era feita a integração com os dados ocupacionais e clínicos. A estimativa do grau de exposição foi baseada em estudos anteriores, realizados através das primeiras 140 avaliações. Nesses foram utilizadas medidas quantitativas de sílica livre na fração respirável no subterrâneo das minas de ouro, em diversas funções, ao longo de mais de 50 anos, além de avaliações qualitativas ${ }^{30}$. Dados de exposições a poeiras minerais ocorridas em outras empresas também foram registrados.

Foram ainda registradas a presença de histórico clínico compatível com doença pulmonar obstrutiva crônica (DPOC), tuberculose pulmonar (TB) ativa ou sequela, hipertensão arterial sistêmica (HAS) e cardiopatias. Em relação à TB, considerou-se ainda a presença de imagens radiológicas típicas da doença ${ }^{31}$.
Para aqueles considerados radiologicamente normais $(0 / 0)$, em que se constatou uma exposição significativa à sílica ${ }^{30}$, assim como os casos duvidosos (0/1) e aqueles com imagens mal definidas ao RX (diagnósticos diferenciais com outras doenças), foi indicada a realização de TCAR. As tomografias foram realizadas em duas clínicas radiológicas, sendo os primeiros $15 \%$ do total de exames realizados na primeira clínica e os demais na segunda. Foram ainda realizadas espirometrias, na maioria dos avaliados, as quais, contudo, não foram objeto deste estudo.

Portanto, o diagnóstico de silicose foi obtido por meio dos procedimentos periciais pré-estabelecidos, que consistiram em: anamnese clínica e ocupacional, exames de imagem- radiografia de tórax padrão OIT e tomografia de tórax, esta última naqueles que apresentaram indicação. Todos os exames foram realizados por ocasião das avaliações.

As variáveis estudadas foram: ano da perícia, idade do trabalhador na data da avaliação, local do posto de trabalho (subterrâneo, superfície, ou ambos), tempo de exposição à sílica, ano de início da exposição e tempo de afastamento.

O subgrupo de maior interesse foi aquele constituído pelos trabalhadores do subterrâneo da mineração de ouro. Por essa razão, em algumas análises, foram excluídos trabalhadores de superfície. Também visava-se evitar interferências de possíveis exposições a poeiras minerais em outras empresas. Para essa finalidade, o critério de exclusão foi o trabalhador com mais de 5 anos de exposição à sílica em outras empresas e expostos em subterrâneo na mineradora deste estudo por período inferior a 5 anos.

Para análise, foi utilizado o programa Statistics Package for Social Sciences (SPSS) versão 15.0. Foram realizadas análises descritivas de frequências, com elaboração de gráficos. Para variáveis de distribuição não normal foi descrita a mediana e o IQR (interquartile range - intervalo interquartil). O estudo faz parte de projeto mais abrangente, aprovado pelos Comitês de Ética em Pesquisa da UFMG (Parecer 098/1999) e do Hospital da Santa Casa de Belo Horizonte (Parecer 742.578).

\section{Resultados}

Foram pesquisados 1.020 prontuários. Das variáveis estudadas houve aproximadamente $7,0 \%$ de falhas de registro, devido à inconsistência ou ausência de dados. As perdas foram nas seguintes variáveis: idade, 79 (7,7\%); local de trabalho, 69 (6,8\%); ano de início da exposição, 73 (7,1\%); duração da exposição, 68 (6,7\%); e tempo de afastamento, 69 (6,9\%).

A maior parte dos indivíduos (835 de 1.020; 81,9\%) foi avaliada entre 2007 e 2009. Todos eram do sexo 
masculino. A idade à época da avaliação apresentou mediana de 53,0 anos (IQR = 15,0), variando de 28 a 96 anos. Quanto à distribuição por faixa etária foi encontrado o seguinte: $58(6,2 \%)$ tinham até 40 anos; $603(64,1 \%)$ de 41 a 60 anos; 264 (28,0\%) de 61 a 80, enquanto apenas 16 (1,7\%) eram maiores que 80 anos.

Quanto ao local de trabalho na mineração, 780 (82,0\%) trabalhavam em subterrâneo, 93 (9,8\%) em superfície e 78 (8,2\%) em ambos.

O ano de início da exposição à sílica se estendeu de 1927 a 1998, com predomínio na década de 1980 que concentrou $42,5 \%$ dos avaliados (Figura 1).

Quanto ao tempo total de duração do trabalho na empresa, a mediana foi de 7,8 anos $(I Q R=11,9)$, com variação de 1 mês a 47,3 anos (Figura 2). Dividindo-se este tempo em faixas, os indivíduos apresentaram a seguinte distribuição: 557 (58,5\%) trabalharam até 10 anos; 320 (33,6\%) de 10,1 a 20 anos e 75 (7,9\%) por um período maior que 20,1 anos. A mediana de afastamento da exposição foi de 19 anos (IQR $=10,1)$, variando de 1 mês a 55 anos. A grande maioria, formada por 823 indivíduos (87,5\%), estava afastada há mais de 10 anos da exposição à sílica. Apenas 66 (7\%) tinham períodos menores que 5 anos de afastamento.

Em relação às radiografias de tórax, a classificação da mediana de profusão de pequenas opacidades foi igual ou maior que 1/0 em 156 casos. Dentre estes havia 26 portadores de grandes opacidades.

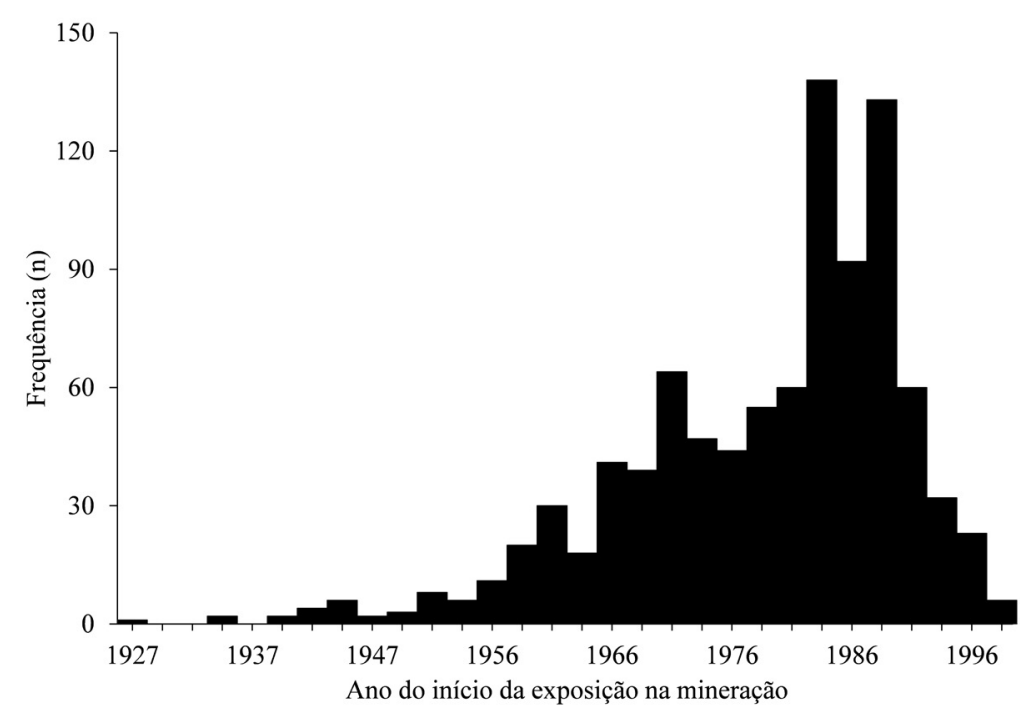

Figura 1 Distribuição do ano de início da exposição à sílica dos avaliados, Nova Lima/MG

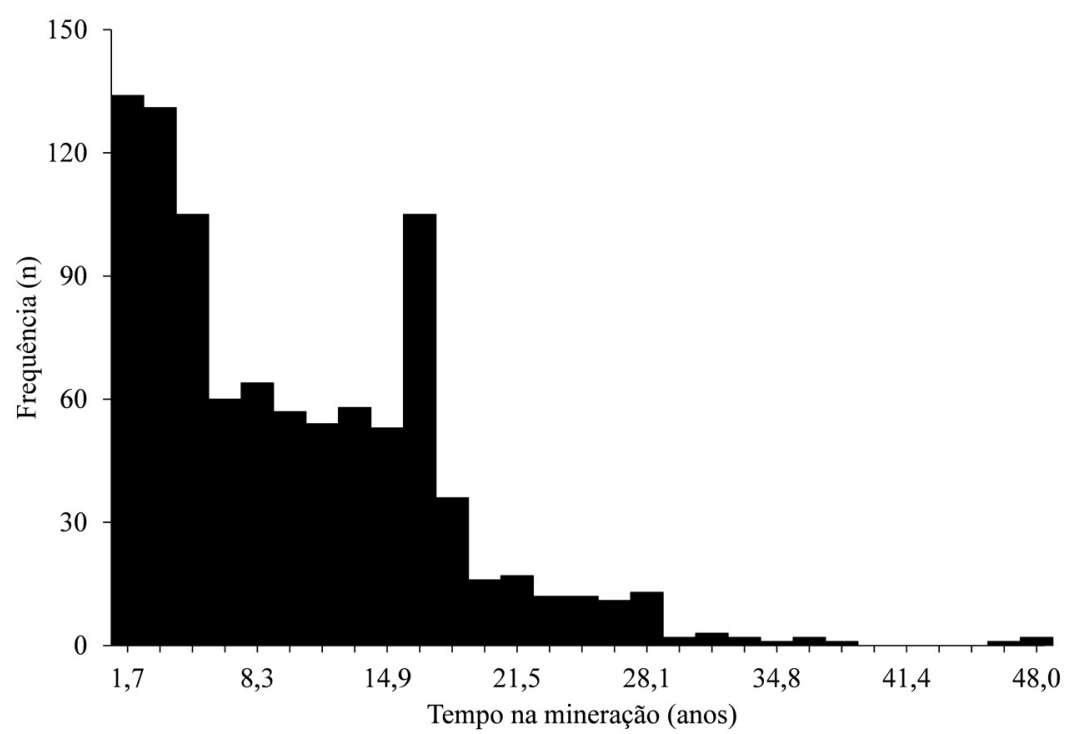

Figura 2 Distribuição do tempo total de duração do trabalho na empresa, Nova Lima/MG 
Tomografias de tórax foram realizadas em 456 indivíduos. Destas, 407 (89,0\%) foram realizadas em portadores de radiografias classificadas como $0 / 0$ ou 0/1, ou seja, buscando-se o diagnóstico de silicose leve ou incipiente, que foi confirmado em 60 casos (14,7\%). Em contrapartida, 49 indivíduos com imagens inicialmente compatíveis com silicose à radiografia, mas com algum grau de dúvida nas imagens ou na integração de dados clínicos e ocupacionais, também foram submetidos à tomografia. Nestes, o diagnóstico da doença foi confirmado em 33 casos $(67,3 \%)$ e afastado em $16(32,6 \%)$, os quais podem ser considerados como falso-positivos radiológicos. Destaca-se que apenas as radiografias de profusão correspondente à categoria 1 apresentaram falso-positivos à tomografia.

Considerando-se conjuntamente resultados de radiografias e tomografias, o diagnóstico de silicose foi realizado em 200 (19,7\%) de 1.014 indivíduos. Em seis não foram recuperados registros de exames de imagem.

Analisando-se a informação do diagnóstico de silicose com relação ao local de trabalho na mineração (subterrâneo, superfície, ou ambos), do total de 200 portadores da doença, 196 exerciam função em subterrâneo e 4 exerciam função em ambos. Nenhum caso foi encontrado em trabalhadores que tiveram atividades exclusivamente na superfície das minas.

Como todos os casos de silicose apresentavam exposição no subterrâneo, a partir deste ponto, foram excluídos das análises os 93 indivíduos que trabalharam apenas na superfície, assim como os $69 \mathrm{sem}$ identificação do local de exposição, restando 858. Destes, encontrou-se que 24 tiveram exposição à sílica em outras empresas por tempo maior que na mineração (5 anos ou mais em outras empresas), de acordo com o critério estabelecido no estudo. Visando minimizar interferência destas exposições externas nas análises de interesse (avaliação temporal e aspectos de dose-resposta da ocorrência de silicose na mineração), esses 24 indivíduos foram excluídos. Excluiu-se ainda um avaliado cujo diagnostico foi de asbestose (exposto em empresa de cimento-amianto). Dessa forma, as análises a seguir referem-se ao grupo restante, formado por 833 pessoas.

No grupo dos 833 indivíduos, restaram 192 dos 200 portadores de silicose. Ou seja, entre os 24 indivíduos excluídos, havia oito casos de silicose cujas exposições ocorreram predominantemente em outras empresas. Nestes últimos, a exposição fora da mineração de ouro variou de 6,3 a 22,3 anos (mediana de 8,8 e IQR $=12,8$ anos).

A Tabela 1 apresenta a frequência das 12 subcategorias radiológicas. Por motivo de ordem prática, a subcategoria $0 /$ - foi incluída na $0 / 0$, assim como a $3 /+$ foi incluída na $3 / 3$. Nessa distribuição não foram considerados os resultados de tomografias. Os portadores de grandes opacidades foram registrados separadamente. O tempo de exposição em subterrâneo foi dividido em quatro faixas de duração (até 5 anos; de 5,1 a 10 anos; de 10,1 a 20 anos e maior que 20 anos). $O$ maior subgrupo estava na faixa mais baixa de exposição (até 5 anos), composto por 311 indivíduos com classificação radiológica 0/0. Nesse subgrupo concentraram-se aqueles nos quais a tomografia não foi considerada necessária, dado a forte característica dose-resposta da doença. Ressalta-se que, entre esses, 164 tinham menos de 2 anos de exposição. Nota-se que, a partir da subcategoria 3/2, todos tinham tempo maior que 10 anos.

Tabela 1 Profusão radiológica de pequenas opacidades em 12 subcategorias e presença de grandes opacidades (de acordo com o padrão OIT) segundo a duração da exposição em subterrâneo, Nova Lima/MG, 1995-2011

\begin{tabular}{|c|c|c|c|c|c|}
\hline \multirow{3}{*}{ Profusão } & \multirow{3}{*}{ Frequência } & \multicolumn{4}{|c|}{ Tempo de exposição (anos) } \\
\hline & & $0-5$ & $5,1-10$ & $10,1-20$ & Maior que 20,1 \\
\hline & & $n(\%)$ & $n(\%)$ & $n(\%)$ & $n(\%)$ \\
\hline $0 / 0$ & 601 & $311(51,7)$ & $124(20,6)$ & $145(24,1)$ & $21(3,5)$ \\
\hline $0 / 1$ & 82 & $23(28,0)$ & $16(19,5)$ & $35(42,7)$ & $8(9,8)$ \\
\hline $1 / 0$ & 30 & $1(3,3)$ & $3(10,0)$ & $24(80,0)$ & $2(6,7)$ \\
\hline $1 / 1$ & 36 & $3(8,3)$ & $10(27,8)$ & $21(58,3)$ & $2(5,6)$ \\
\hline $1 / 2$ & 14 & $2(14,3)$ & $1(7,1)$ & $8(57,1)$ & $3(21,4)$ \\
\hline $2 / 1$ & 10 & $0(0)$ & $3(30,0)$ & $6(60,0)$ & $1(10,0)$ \\
\hline $2 / 2$ & 18 & $2(11,1)$ & $3(16,7)$ & $12(66,7)$ & $1(5,6)$ \\
\hline $2 / 3$ & 7 & $1(14,3)$ & $0(0,0)$ & $6(85,7)$ & $0(0)$ \\
\hline $3 / 2$ & 4 & $0(0,0)$ & $0(0,0)$ & $3(75,0)$ & $1(25,0)$ \\
\hline $3 / 3$ & 5 & $0(0)$ & $0(0,0)$ & $4(80,0)$ & $1(20,0)$ \\
\hline Grandes opacidades & 26 & $2(7,7)$ & $2(7,7)$ & $18(69,2)$ & $4(15,4)$ \\
\hline Total $(\%)$ & $833(100)$ & $344(41,3)$ & $163(19,6)$ & $282(33,8)$ & $44(5,3)$ \\
\hline
\end{tabular}


Ao se distribuir a prevalência da silicose (considerando-se diagnósticos por radiografia e/ou tomografia) obtido no grupo de 833 indivíduos, de acordo com faixas de tempo da exposição no subterrâneo, obtiveram-se resultados demonstrados na Figura 3.

No mesmo grupo, analisando-se a prevalência de silicose, do ponto de vista temporal, segundo a década de início da exposição à sílica no subterrâneo da mineração, obtiveram-se resultados demonstrados na Figura 4.

Em relação às formas mais graves de silicose, representadas pela presença de grandes opacidades e profusão grau 3 de pequenas opacidades, encontrou-se que: os que apresentavam grandes opacidades $(n=26)$ tiveram o ano de início da exposição até no máximo 1985 e mediana do tempo de exposição de 15,0 anos. Os classificados na categoria $3(\mathrm{n}=9)$ iniciaram a exposição até no máximo 1979 e tiveram a mediana de tempo de exposição de 16,0 anos.

Quanto ao encontro de outras doenças com base na história clínica, avaliando-se todo o grupo pesquisado, constituído pelos 1.020 indivíduos, destaca-se a TB pregressa, identificada a partir de dados clínicos e/ou radiológicos em 72 (7,0\%) e a DPOC, identificada pela história clínica, em 73 (7,1\%). Além disso, foram encontrados 314 portadores de HAS e 65 cardiopatas de acordo com dados clínicos. Houve ainda história clínica e imagem característica de neoplasia de pulmão em três indivíduos. Outros 141 informaram terem sofrido acidentes típicos na empresa.

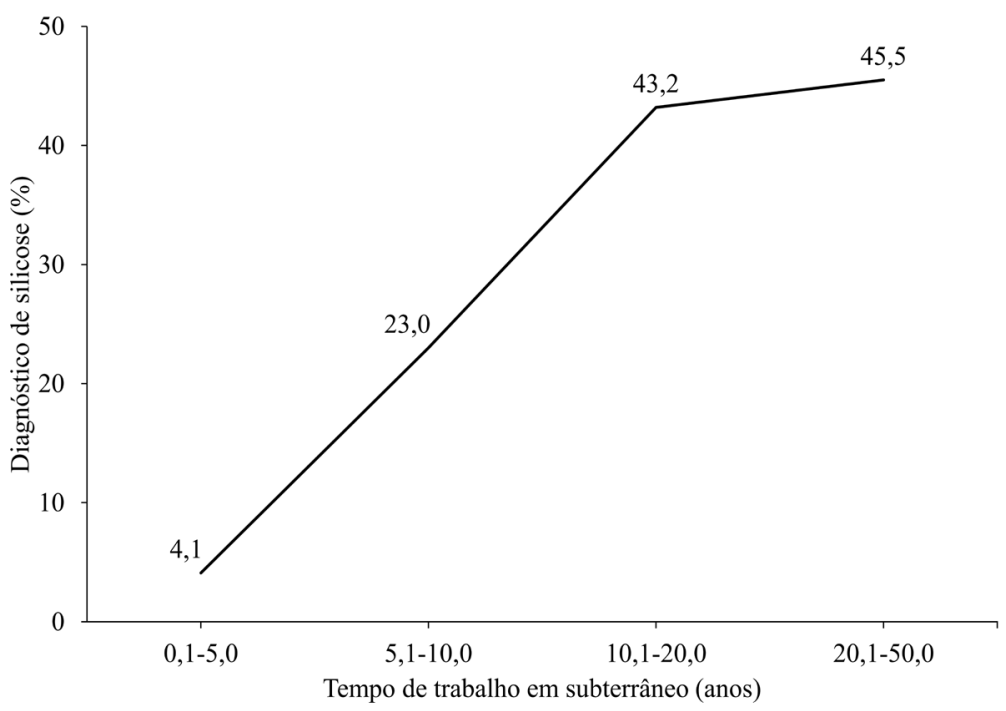

Figura 3 Prevalência da silicose em faixas de tempo da exposição no subterrâneo, 833 indivíduos, Nova Lima/MG

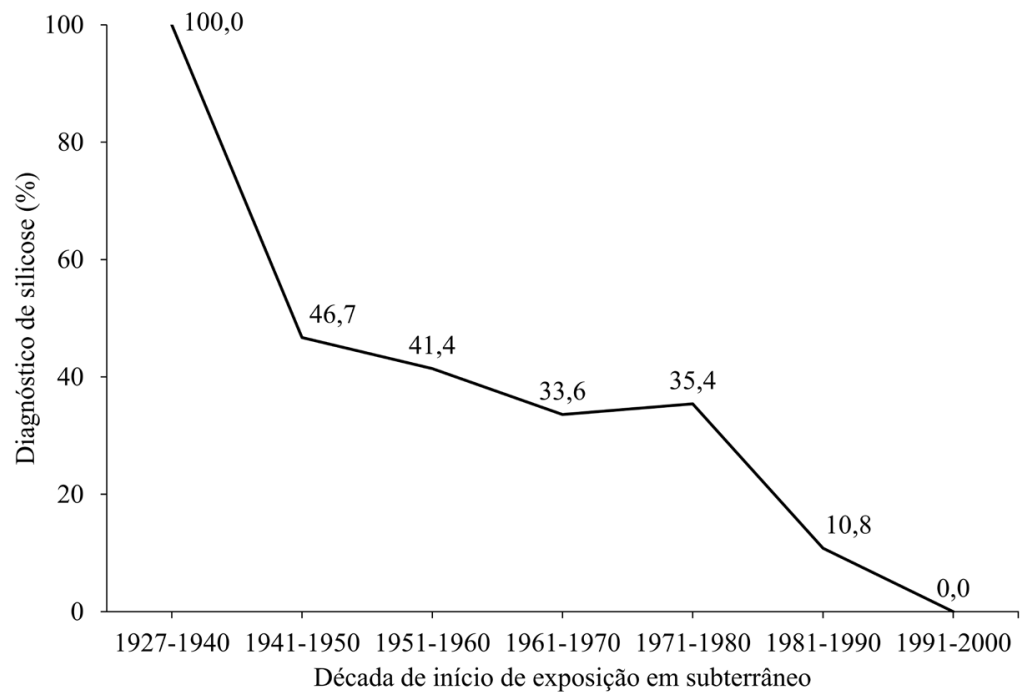

Figura 4 Prevalência de silicose segundo a década de início da exposição à sílica no subterrâneo da mineração, 833 indivíduos, Nova Lima/MG 


\section{Discussão}

Notou-se uma queda expressiva na prevalência de silicose no setor de mineração de ouro no período estudado. Uma vez que a doença é sabidamente dose-dependente, já era esperado que houvesse diminuição do número de casos, em consequência da redução nos níveis de exposição ocorrida ao longo dos anos ${ }^{30,32}$. No entanto, até o momento, não havia registros publicados de dados como estes.

Sabe-se que a dose cumulativa de poeira inalada é formada por dois componentes principais: a duração da exposição e a concentração de poeira ${ }^{1,13}$. No componente representado pela duração da exposição, com relação ao tempo de trabalho na mineração estudada (Figura 2), observou-se que a maior parte dos trabalhadores $(58,5 \%)$ teve tempo de exposição até 10 anos, sendo que $75,5 \%$ não atingiram o tempo mínimo previsto em lei para a aposentadoria especial de trabalhadores em subterrâneo de minas, que é de 15 anos. Alguns alternaram períodos de trabalho na superfície com períodos de trabalho no subterrâneo. É perceptível a presença de trabalhadores com períodos muito curtos de trabalho na mineração de ouro, como os $38,9 \%$ que trabalharam por menos de 5 anos.

Ainda em relação ao componente de duração da exposição, observou-se um claro gradiente dose-resposta, ou seja, prevalências bem mais baixas da silicose em períodos mais curtos de exposição (Figura 3).

Ressalta-se que vários trabalhadores avaliados tiveram exposições respiratórias em outras empresas, incluindo outras exposições à sílica, alguns até mesmo em minerações diferentes. Por essa razão excluíram-se das análises 24 indivíduos cuja exposição na mineração foi proporcionalmente menor que exposições externas. Entre estes, havia oito portadores de silicose que, embora a exposição na mineração possa ter contribuído para o desenvolvimento da doença, tinham indícios de maior exposição em outras empresas.

A variável "década de início da exposição" em subterrâneo demonstrou ser um bom indicador da exposição a poeiras, como mostra a Figura 4. Neste ponto, uma limitação foi a provável sub-representação de indivíduos mais idosos, ou seja, aqueles que iniciaram suas atividades em mineração em décadas mais antigas. Esses indivíduos podem já ter falecido, talvez por influência da própria silicose, que estaria, portanto, subestimada. Apesar dessa limitação, evidenciou-se que houve nítida queda da prevalência de doentes com o passar do tempo.

Já em relação aos que iniciaram a exposição em subterrâneo após 1990, portanto expostos a níveis mais baixos de sílica, havia 15 indivíduos cujo tempo de afastamento foi menor que 5 anos. Nestes, há ainda que se considerar que o tempo de observação até o momento das avaliações pode ter sido relativamente curto para se afirmar sobre o não desenvolvimento da silicose. Serão necessárias futuras avaliações para complementar essa informação. Em contrapartida, para maior parte dos avaliados, diante do longo tempo de afastamento da exposição (mediana 19 anos), torna-se pouco provável que surjam novos casos de silicose além dos já diagnosticados ${ }^{33}$. Estudo de coorte com mais de 2.000 mineiros de ouro na África do Sul evidenciou média de 7,4 anos (desvio-padrão de 5,5 ) para surgimento de casos de silicose após afastados da exposição à sílica. Nesse estudo, o critério diagnóstico por imagem foi a profusão $1 / 1$, ainda mais criterioso do que a profusão 1/0 adotada no Brasil ${ }^{33}$.

É fato que, ao longo dos anos, houve maior rigor dos meios de fiscalização, o que forçosamente promoveu melhorias dos ambientes e equipamentos de trabalho. Houve reestruturação dos sistemas de ventilação e exaustão, umidificação dos processos de exploração de minério dentro das minas, substituição de perfuratrizes manuais pelo uso de equipamentos com cabine fechada para o operador, assim como melhor qualidade e controle no uso de equipamento de proteção individual respiratórios. Tais medidas reduziram a concentração de poeira, conforme já avaliado em estudo anterior ${ }^{30}$ que analisou os níveis de exposição entre 1933 até 1986, considerando-se as diversas tarefas exercidas em subterrâneo, variando de $12,45 \mathrm{mg} / \mathrm{m}^{3}$ a $0,012 \mathrm{mg} / \mathrm{m}^{3}$.

Outra importante diferença em relação à concentração de poeira diz respeito ao local de trabalho, se subterrâneo ou superfície. Sabe-se que as tarefas subterrâneas geram níveis bem mais elevados de poeira que as da superfície. Neste estudo não houve confirmação de silicose em indivíduos que tenham trabalhado exclusivamente na superfície.

Entretanto, o estudo atual apresenta limitações nas estimativas dos níveis de exposição, uma vez que nem todas as variáveis que determinam a concentração de sílica inalada foram analisadas. Por exemplo, embora nos prontuários exista registro das funções exercidas pelos trabalhadores e em quais das minas eles trabalharam, essas informações não foram aqui analisadas. Sabe-se que a concentração de poeira difere muito em relação às funções desenvolvidas no subterrâneo e que existe ainda variação dos níveis de poeira de acordo com as condições de cada mina, no decorrer dos anos ${ }^{30}$.

Em relação às demais doenças relacionadas à sílica, embora este estudo não tenha se proposto a avaliá-las, houve registro de casos de TB e DPOC. No 
que diz respeito à TB, encontrou-se 7,0\% de indivíduos com sequelas radiológicas e/ou história clínica dessa doença. Segundo a literatura, portadores de silicose apresentam taxas de TB de 10 a 20 vezes maiores que a população geral ${ }^{7,11}$. Em relação à DPOC não foram analisadas espirometrias, nem exposições ao tabagismo no estudo atual. Portanto a taxa de 7,1\% de portadores de doença pulmonar obstrutiva crônica deve estar subestimada, pois se limitou ao registro de história clínica. Houve ainda registro de diversos casos de doenças cardiovasculares, osteomusculares e traumas que, como não faziam parte dos objetivos do estudo, não seguiram em análise.

Por fim, este estudo evidenciou grande diferença entre os supostos diagnósticos de silicose, que serviram de elemento para instauração das ações judiciais, e aqueles confirmados nas perícias. Apenas em $19,7 \%$ dos indivíduos a doença foi confirmada. Esta grande diferença pode ser atribuída ao desconhecimento da correta técnica radiológica padrão OIT ou falta de treinamento e qualificação na interpretação de radiografias suspeitas por parte dos médicos que fizeram as antigas avaliações. Outros fatores podem incluir dificuldades operacionais e limitações dos equipamentos de aquisição de imagem. Soma-se a isso o fato das antigas avaliações não terem contado com a disponibilidade de tomografias. O melhor desempenho desse método se deve à maior resolução espacial, com detalhamento corte a corte do parênquima e a visualização direta das finas estruturas do interstício pulmonar. É possível ainda que nas avaliações prévias, realizadas por médicos não especialistas, tenha havido influência ou confusão de diagnóstico diferencial com outras pneumopatias, como tuberculose e DPOC, ou mesmo com as finas estruturas do interstício pulmonar ${ }^{34}$.
Destaca-se que a época de início do trabalho pericial houve intensa mobilização do movimento sindical local, com empenho no processo de negociação coletiva, na busca por melhorias das condições de trabalho, e forte estímulo à reinvindicação de reparação de danos, incentivando os trabalhadores a mover ações contra o empregador ${ }^{35}$. Acrescenta-se que havia um ambiente de profunda comoção e mobilização social por responsabilização do empregador e reparação monetária pelas condições inadequadas e agravos à saúde relacionados ao trabalho. A silicose era o mais reconhecido e visível agravo, mas não o único. Esse clima gerou grande demanda de trabalhadores, de seus representantes legais e escritórios de advocacia, por laudos atestando a presença da doença, de forma a dar início a uma ação judicial ${ }^{35}$.

\section{Conclusão}

Foi evidenciada nítida diminuição da ocorrência de silicose no período avaliado. Este achado certamente é reflexo da diminuição dos níveis de poeira ocorrida ao longo dos anos e, provavelmente, decorre da adoção de novas tecnologias e mudanças nos processos de trabalho, apesar de não terem sido analisadas neste estudo todas as variáveis envolvidas na determinação da concentração de poeira na mineração.

É fundamental que tais medidas continuem a ser adotadas neste e em outros ramos de atividade, visando atingir níveis cada vez menores de sílica. Isso irá favorecer a prevenção não só da silicose, mas também de outras doenças relacionadas à sílica reconhecidas na literatura, como tuberculose, DPOC, doenças autoimunes e câncer de pulmão.

\section{Contribuições de autoria}

Silva LL, Lima LPC, Carneiro APS contribuíram na concepção e planejamento, na análise e interpretação dos dados, na elaboração do texto, na revisão crítica do conteúdo e na aprovação da versão final do manuscrito. Silveira AM, Torre DNPD, Silva FCL contribuíram na análise e interpretação dos dados, na elaboração do texto, na revisão crítica do conteúdo e na aprovação da versão final do manuscrito. Barbosa CC, Machado AD, Mosci AS contribuíram na concepção e planejamento, na revisão crítica do conteúdo, e na aprovação da versão final do manuscrito.

\section{Referências}

1. Leung CC, Yu IT, Chen W. Silicosis. The Lancet [periódicos na Internet]. 2012 [acesso em 2018 abr 27];379(9830):2008-18. Disponível em: https:// www.thelancet.com/journals/lancet/article/PIIS01 40-6736(12)60235-9/fulltext DOI: 10.1016/S01406736(12)60235-9
2. Carneiro APS, Algranti E. Silicose e doenças não malignas relacionadas à sílica. In: Santos UP, organizador. Pneumologia ocupacional ilustrada: fotos e fatos. Rio de Janeiro: Atheneu; 2013. p. 81-9.

3. American Thoracic Society. Adverse effects of crystalline silica exposure. Am J Respir 
Crit Care Med [periódicos na Internet]. 1997 [acesso em 2018 abr 27];155(2):761-65. Disponível em: https://www.atsjournals.org/doi/ abs/10.1164/ajrccm.155.2.9032226 DOI: 10.1164/ ajrccm.155.2.9032226

4. Fubini B. Surface chemistry and quartz hazard. Ann Occup Hyg [periódicos na Internet]. 1998 [acesso em 2018 abr 27];42(8):521-30. Disponível em: https://academic.oup.com/ annweh/article/42/8/521/148104 DOI: 10.1093/ annhyg/42.8.521

5. Turci F, Cristina PC, Leinardi R, Tomatis M, Pastero L, Garry D, et al. Revisiting the paradigm of silica pathogenicity with synthetic quartz crystals: the role of crystallinity and surface disorder. Part Fibre Toxicol [periódicos na Internet]. 2016 [acesso em 2018 abr 27];13(1):32. Disponível em: https:// particleandfibretoxicology.biomedcentral.com/ articles/10.1186/s12989-016-0136-6 DOI: 10.1186/ s12989-016-0136-6

6. Rose C. Silicosis [Internet]. 2016 [acesso em 2018 abr 27]. Disponível em: https://www.uptodate.com/ contents/silicosis

7. teWaterNaude JM, Ehrlich RI, Churchyard GJ, Pemba L, Dekker K, Vermeis M, et al. Tuberculosis and silica exposure in South African gold miners. Occup Environ Med [periódicos na Internet]. 2006 [acesso em 2018 abr 27];63(3):187-92. Disponível em: http://oem.bmj.com/content/63/3/187 DOI: 10.1136/oem.2004.018614

8. Pollard KM. Silica, silicosis and autoimmunity. Front Immunol [periódicos na Internet]. 2016 [acesso em 2018 abr 27];7:97. Disponível em: https://www.ncbi.nlm.nih.gov/pmc/articles/ PMC4786551/ DOI: 10.3389/fimmu.2016.00097

9. Hnizdo E, Vallyathan V. Chronic obstructive pulmonary disease due to occupational exposure to silica dust: a review of epidemiological and pathological evidence. Occup Environ Med [periódicos na Internet]. 2003 [acesso em $2018 \mathrm{abr}$ 27];60(4):237-43. Disponível em: http://oem.bmj. com/content/60/4/237 DOI: 10.1136/oem.60.4.237

10. IARC. Silica dust, crystalline, in the form of quartz or cristobalite [Internet]. Lyon (FR): IARC; 2012 [acesso em 2018 abr 27]. Disponível em: https:// www.ncbi.nlm.nih.gov/books/NBK304370/

11. NIOSH. Health effects of occupational exposure to respirable crystalline sílica [Internet]. Atlanta: NIOSH; Center for Disease Control and Prevention Publication; 2002 [acesso em 2018 abr 27]. p. 129. Disponível em: https://www.cdc.gov/niosh/ docs/2002-129/pdfs/2002-129.pdf

12. Buchanan D, Miller BG, Soutar AC. Quantitative relations between exposure to respirable quartz and risk of silicosis. Occup Environ Med [periódicos na Internet]. 2003 [acesso em $2018 \mathrm{abr}$ 27];60(3):159-64. Disponível em: http://oem.bmj. com/content/60/3/159 DOI: 10.1136/oem.60.3.159

13. Churchyard GJ, Ehrlich R, teWaterNaude JM, Pemba L, Dekker K, Vermeijs M, et al. Silicosis prevalence and exposure-response relations in
South African goldminers. Occup Environ Med [periódicos na Internet]. 2004 [acesso em 2018 abr 27];61(10):811-6. Disponível em: http:// oem.bmj.com/content/61/10/811 DOI: 10.1136/ oem.2003.010967

14. Corbett EL, Mozzato-Chamay N, Butterworth AE, De Cock KM, Williams BG, Churchyard GJ, et al. Polymorphisms in the tumor necrosis factoralpha gene promoter may predispose to severe silicosis in black South African miners. Am J Respir Crit Care Med [periódicos na Internet]. 2002 [acesso em 2018 abr 27];165(5):690-3. Disponível em: https://www.atsjournals.org/doi/ abs/10.1164/ajrccm.165.5.2010050 DOI: 10.1164/ ajrccm.165.5.2010050

15. Organização Internacional do Trabalho (OIT). Guidelines for the use of the ILO International Classification of Radiographs of pneumoconiosis. Geneva: International Labour Organization; 2011.

16. Bhawna S, Ojha UC, Kumar S, Gupta R, Gothi D, Pal RS. Spectrum of high resolution computed tomography findings in occupational lung disease: experience in a Tertiary Care Institute. J Clin Imaging Sci [periódicos na Internet]. 2013 [acesso em 2018 abr 27];3(4):64. Disponível em: http://www.clinicalimagingscience.org/text. asp?2013/3/1/64/124097 DOI: 10.4103/21567514.124097

17. Weng D, Jin C, Yan B, Xu G, Jin B, Xia S, et al. The value of high resolution computed tomography in the diagnostics of small opacities and complications of silicosis in mine machinery manufacturing workers, compared to radiography. J Occup Health [periódicos na Internet]. 2008 [acesso em 2018 abr 27];50(5):400-5. Disponível em: https://pdfs.semanticscholar.org/f44a/9f05b6d8 22266bdef3971a171e9713f51b5b.pdf

18. Talini D, Paggiaro PL, Falaschi F, Batolla L,Carrara M, Petrozzino M, et al. Chest radiography and high resolution computed tomography in the evaluation of workers exposed to silica dust: relation with functional findings. Occup Environ Med [periódicos na Internet]. 1995 [acesso em $2018 \mathrm{abr}$ 27];52(4):262-7. Disponível em: https://www.ncbi. nlm.nih.gov/pmc/articles/PMC1128205/

19. Bacchus L, Shah RD, Chung JH, Crabtree TP, Heitkamp DE, Iannettoni MD, et. al. ACR Appropriateness Criteria ${ }^{\circledR}$ occupational lung diseases. J Thoracic Imaging [periódicos na Internet]. 2016 [acesso em 2018 abr 27];31(1):W13. Disponível em: https://journals.lww.com/ thoracicimaging/pages/articleviewer.aspx?year $=2$ 016\&issue $=01000 \&$ article $=00011 \&$ type $=$ abstract DOI: 10.1097/RTI.0000000000000194

20. Ribeiro FSN, Camargo EA, Algranti E, Wunsch FV. Exposição ocupacional à sílica no Brasil no ano de 2001. Rev Bras Epidemiol [periódicos na Internet]. 2008 [acesso em 2018 abr 27];11(1):8996. Disponível em: http://www.scielo.br/pdf/rbepid/ v11n1/08.pdf

21. Algranti E, De Capitani EM, Carneiro APS, Saldiva PHN, Mendonça EMC. Doenças respiratórias 
relacionadas com o trabalho. In: Mendes R, organizador. Patologia do trabalho. 3. ed. Rio de Janeiro: Atheneu; 2013. p. 1229-90.

22. Ministério da Saúde. Fundação Nacional de Saúde. Manual de normas para controle das pneumoconioses: silicose, pneumoconiose dos trabalhadores do carvão e pneumoconioses por poeiras mistas. Brasília, DF: MS; 1997.

23. Carneiro APS, Campos LO, Gomes MFCF, Assunção AA. Perfil de 300 trabalhadores expostos à sílica atendidos ambulatorialmente em Belo Horizonte. J Pneumologia [periódicos na Internet]. 2002 [acesso em 2018 abr 27];28(6):329-34. Disponível em: http://www.scielo.br/pdf/jpneu/ v28n6/a06v28n6.pdf

24. Barbosa MSA, Carneiro APS, Maciel JGFS, Moronte EA, La Rocca PF, Santos ARM. Silicose em trabalhadores de quartzito da região de São Thomé das Letras -Minas Gerais: dados iniciais indicam um grave problema de saúde pública. Rev Bras Saúde Ocup [periódicos na Internet]. 2011 [acesso em 2018 abr 27];36(123):177-84. Disponível em: http://www.scielo.br/pdf/rbso/v36n123/ a18v36n123.pdf

25. Ferreira LR, Pinheiro TMM, Siqueira AL, Carneiro APS. A silicose e o perfil dos lapidários de pedras semipreciosas em Joaquim Felício, Minas Gerais, Brasil. Cad Saúde Pública [periódicos na Internet]. 2008 [acesso em 2018 abr 27];24(7):1517-26. Disponível em: http://www.scielo.br/pdf/csp/ v24n7/06.pdf

26. Brasil. Departamento Nacional da Produção Mineral. Higiene das minas de ouro. Silicose e outras doenças dos mineiros da Passagem, Minas Gerais. Rio de Janeiro; 1942. Boletim n. 53.

27. Brasil. Departamento Nacional da Produção Mineral. Divisão de Fomento da produção mineral. Silicose e silico-tuberculose. Rio de Janeiro; 1951. Boletim n. 89. 56p.

28. Mineração Morro Velho. Morro Velho: história, fatos e feitos. Nova Lima: Mineração Morro Velho; 1996. 205p.
29. Lima DA, Lorenzo GV, Amaral LS, Sampaio MR Relatório técnico das condições de segurança, higiene e medicina do trabalho e trabalho educativo na Mineração Morro Velho S/A. Belo Horizonte: Fundacentro CEMG; 1991-1992.

30. Carneiro APS, Barreto SM, Siqueira AL, La Rocca PF. Índice de exposição à sílica na atividade de mineração de ouro. Rev Saúde Pública [periódicos na Internet]. 2006 [acesso em 2018 abr 27];40(1):83-91. Disponível em: http://www.scielo. br/pdf/rsp/v40n1/27120.pdf DOI: 10.1590/S003489102006000100014

31. Fraser RS, Muller NL, Colman N, Paré PD. Mycobacteria In: Fraser RG, Peter Paré JA, editores. Diagnosis of diseases of the chest. 4. ed. Philadelphia: WB Saunders Company; 1999. V. 2. p. 799-873.

32. Lima DA, Lorenzo GV, Amaral LS, Sampaio MR. Relatório técnico das condições de segurança, higiene e medicina do trabalho e trabalho educativo na Mineração Morro Velho S/A. Belo Horizonte: Fundacentro CEMG; 1991-1992.

33. Hnizdo E, Sluis-Cremer G. Risk of silicosis in a cohort of White South African gold miners. Am J Ind Med [periódicos na Internet]. 1993 [acesso em 2018 abr 27];24(4):447-57. Disponível em: https://onlinelibrary.wiley.com/doi/pdf/10.1002/ ajim.4700240409 DOI: 10.1002/ajim.4700240409

34. Gaik C, Ooi GC, Tsang KW, Cheung TF, Khong PL, Ho IW, et al. Silicosis in 76 men: qualitative and quantitative CT evaluation-clinical-radiologic correlation study. Radiology [periódicos na Internet]. 2003 [acesso em 2018 abr 27];228(3):81625. Disponível em: https://pubs.rsna.org/ doi/10.1148/radiol.2283020557 DOI: 10.1148/ radiol.2283020557

35. Almeida JR, Martins RTO. Da poeira à mobilização: o movimento dos silicóticos da mineração Morro Velho. Cadernos de Pesquisa do CDHIS [periódicos na Internet]. 2008 [acesso em 2018 abr 27]; 39(21):55-61. Disponível em: http://www.seer.ufu. br/index.php/cdhis/article/view/2254/1848 DOI: 10.14393/cdhis.v1i39.2254 